\title{
A Design-Centred Framework for Social Human-Robot Interaction
}

\author{
Christoph Bartneck ${ }^{1}$ and Jodi Forlizzi ${ }^{2}$ \\ ${ }^{1}$ Department of Industrial Design, Eindhoven University of Technology, the Netherlands \\ ${ }^{2}$ School of Design and HCII, Carnegie Mellon University, Pittsburgh, PA, USA \\ christoph@bartneck.de, forlizzi@cs.cmu.edu
}

\begin{abstract}
Robots currently integrate into our everyday lives, but little is known about how they can act socially. In this paper we propose a definition of social robots and describe a framework that classifies properties of social robots. The properties consist of form, modality, social norms, autonomy, and interactivity. Finally, we provide broad guidelines for the design of social robots.
\end{abstract}

\section{Introduction}

Robotics is a broad discipline. The United Nations (UN), in a recent robotics survey, groups robotics into three major categories: industrial robotics, professional service robotics, and personal service robotics (UN, 2002). Industrial robots have historically represented the vast majority of robotic development, with many deployed in the automotive industry, beginning with the entire automation of a Nissan plant in the 1990s (MetalForming, 1997). Professional service robots are the subject of a much less practiced field, but it is quickly growing. These robots work in domains inaccessible to people, such as navigating abandoned mines and cleaning up nuclear waste. Personal service robots have the highest expected growth rate. They are estimated to grow from 176,500 in 2001 to 2,021,000 in 2005 — an amazing growth rate (UN, 2002)! Personal service robots assist people directly in domestic and institutional settings. Many robots interact with people who have no special skills or training to operate the robot. The design and interaction of these robots will be critical, and raises a number of research and design challenges.

These personal service robots are now robust enough to be deployed in industrial, institutional, and domestic settings and they have the potential to be greatly beneficial to humankind. However, how these robots should behave and interact with humans — act socially — remains largely unclear. For example, a robot might be perceived as having intentional behavior that is usually only ascribed to living things. Researchers and designers have only just begun to understand these critical issues.

In this paper, we attempt to define social robots and present a framework to classify properties of social robots. The properties consist of form, modality, social norms, autonomy, and interactivity. Based on the framework, we provide broad guidelines for the design of social robots. We envision that more specific guidelines will be generated as robots are designed and built to address specific application areas.

\section{Background and Related Work}

A number of theoretical approaches have been developed to characterize the emerging area of human-robot interactions. A first review of social robots is now available (Fong, Nourbakhsh, \& Dautenhahn, 2003). Several of these approaches are driven by technological advances that enable more natural and lifelike human-robot interactions (Breazeal, 2003; Brooks, 2002).

Guidelines for how the social nature of the robot is expressed can be taken from social literature on human social interaction. For example, Goffman described the exchange between two people as taking the form of either a focused or unfocused interaction (Goffman, 1963). A focused interaction is initiated by an encounter such as eye contact, or a statement made in a particular tone of voice. When the other responds, the interaction moves into the engagement phase, characterized by mutual glances, proximity of one speaker to the other, and gesture. Condon classified communication between two people as taking a number of forms: phatic communication (small talk), recordtransmitting or instrumental communication (focusing on results), affective communication, catharsis, and even magic and ritual (Condon, 1965). Hall described the concept of adumbration as the indications preceding or surrounding formal communications that enable people to engage in a mutual exchange (Hall, 1964). These are classified as vocal, mechanical, mobile, or terrestrial.

To communicate socially, robots might mimic human emotion. Several proposals for an "architecture of emotion" have been made (Ortony, Clore, \& Collins, 1988; Trappl, Petta, \& Payr, 2003) and their application discussed (Bartneck, 2002). Several researchers have successfully applied some of these theories and concepts to the design of a variety of social robots (RoboticPerformanceCompany, 2004).

\section{What is a social robot?}

Definitions of robots and social robots are continuously under debate. The notion of what a robot is has migrated since its first appearance 1920 in Karel Capek's play (Capek, 2001). Here, the term "robot" was coined, and robots were given a negative connotation as evil machines that would subsume mankind. The Robot Institute of America defines a 
robot as "A reprogrammable, multifunctional manipulator designed to move material, parts, tools, or specialized devices through various programmed motions for the performance of a variety of tasks" and the International Standard Organization (ISO) in ISO 8373 defines a robot as "An automatically controlled, reprogrammable, multipurpose, manipulator programmable in three or more axes, which may be either fixed in place or mobile for use in industrial automation applications."

These definitions are clearly targeted to autonomous or semi-autonomous industrial robots and do not take interaction with humans into account aside from the attached safety regulations, such as ANSI/RIA R15.06-1999 and ISO12100. The growth in personal service robots necessitates a definition for the kinds of robots that work with people. The International Federation of Robotics (IFR) has adopted a preliminary definition of Service Robots as "A robot which operates semi or fully autonomously to perform services useful to the well being of humans and equipment, excluding manufacturing operations."

What is not explicitly mentioned in this definition is the interaction between people and robots, which is mentioned by Engelhardt (Engelhardt \& Edwards, 1992) in his definition of service robots as "Systems that function as smart, programmable tools, that can sense, think, and act to benefit or enable humans or extend/enhance human productivity." This definition speaks more about human productivity and less about social interaction, the goals of which are not always productivity. For example, entertainment robots including products such as the Sony Aibo (Sony, 1999) are not exceedingly productive but are still very valuable to their owners. We would like to propose the following definition of a social robot:

A social robot is an autonomous or semi-autonomous robot that interacts and communicates with humans by following the behavioral norms expected by the people with whom the robot is intended to interact.

This definition implies that a social robot has a physical embodiment. Screen characters or any kind of virtual agent would be excluded by this definition. Recently, a class of robots have been developed that use a screen to display the robot's head (RoboticPerformanceCompany, 2004). Because the screen-based head sets an expectation for and a locus of interaction, it can be considered to be a social robot.

Autonomy is a requirement for a social robot. A semiautonomous robot can be defined as social if it communicates an acceptable set of social norms. A completely remote controlled robot cannot be considered to be social since it does not make decisions by itself. It is merely an extension of another human.

Communication and interaction with humans is a critical point in this definition. Therefore, robots that only interact and communicate with other robots would not be considered to be social robots. The interaction is likely to be cooperative, but is not limited to it. Also uncooperative behavior can be considered social in certain situations. The robot could, for example, exhibit competitive behavior within the framework of a game. The robot could also interact with a minimum or no communication. It could, for example, hand tools to an astronaut working on a space station (Goza, Ambrose, Diftler, \& Spain, 2004).

In our definition, being social is bound to understanding and in some cases, mimicking human activity and the surrounding society and culture, which shapes social values, norms and standards. For example, a robotic butler should comply with established rules of good service. It should anticipate, be reliable, and most of all, be discreet. However, the precise activities are likely to vary between cultures since social values, norms and standards differ between cultures (Hofstede, 1984). With this definition in place we can now turn to a framework that classifies properties of social robots.

\section{The Framework}

Our framework takes a design-centered perspective, viewing social robots as products that facilitate co-experience and social interaction (Forlizzi \& Battarbee, 2004). The framework also focuses on the notion of designed form. Design approaches form as the total expression of the product - not just how something appears, but the whole experience of the interacting with the product. Form includes a product's physical manifestation, materials, and behavioral qualities (DiSalvo, Gemperle, Forlizzi, \& Montgomery, 2003). Designers use form to balance the needs of people, the capabilities of technology, and the context of use into a single product.

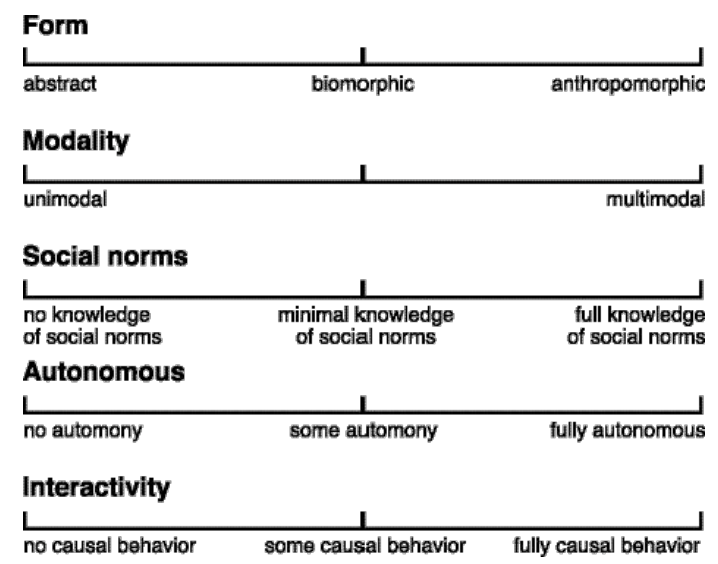

Figure 1. Framework for classifying social robots.

Our framework (Figure 1) contains the following properties:

\section{a. form}

For the purposes of this investigation, we group form (shape, materials, and behavioral qualities) into three categories that suggest social behavior: abstract, biomorphic 
(mimicking a lifelike object), or anthropomorphic (mimicking a human).

\section{b. modality}

We define modality as the number of communication channels engaged. The scale ranges from unimodal to multimodal, and can encompass the visual, auditory, haptic, olfactory and even gustatory channels.

\section{c. social norms}

Social norms assume that much of our behavior is influenced by how other members of our social group behave. As social norms can be defined by the interactions between people, we assert that they can be defined by the interactions between people and robots. We define social norms as a continuum ranging from the robot exhibiting no apparent social norms - for instance, a Furby (Hasbro, 2004), to exhibiting apparent reciprocal social norms. To our knowledge, scientists and designers have yet to realize this goal.

\section{d. autonomy}

We define autonomy as having the technological capabilities to act on behalf of humans without direct input from humans. Autonomy is expressed as a continuum ranging from no autonomy, to some autonomy, to fully autonomous.

\section{e. interactivity}

We define interactivity as having the potential to exhibit causal behavior - that is, to respond in reaction to interaction with a human. Causal behavior is expressed as a continuum ranging from no causal behavior, to some causal behavior, to fully causal behavior.

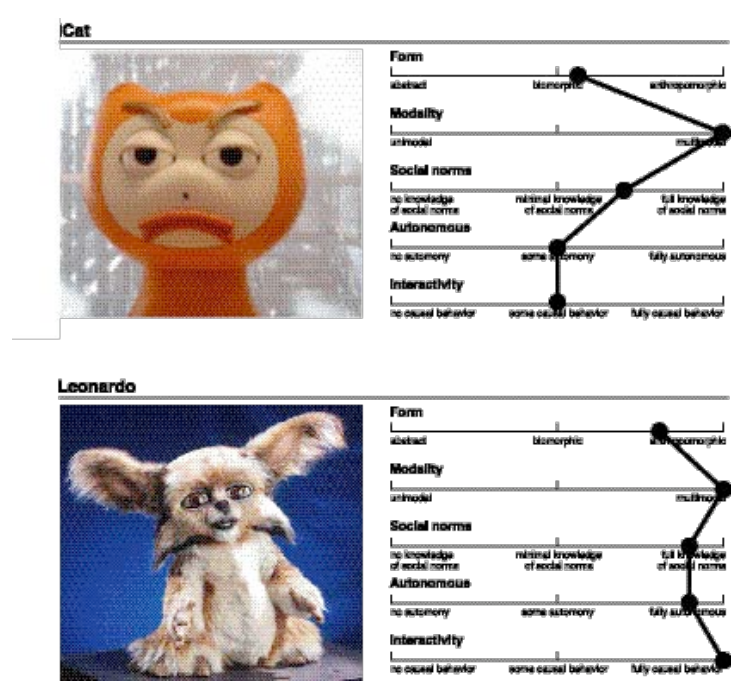

Figure 2. Example classifications using the framework.

To exemplify how the framework works in practice, we have classified a few examples presented at our CHI2004 Social
Robot Workshop (figure 2). These include iCat (Breemen, 2004) and Leonardo (Hoffman \& Breazeal, 2004).

\section{Applications of the Framework}

The development of social robots will continue to escalate at an unprecedented pace. Through our research and design work, we have developed numerous ideas for the design of social robots in a variety of contexts. In this section, we propose the following design guidelines for social robots:

\section{The form of the social robot should match its abilities.}

The shape, size, and material qualities of a social robot should match the task it is designed for to avoid false expectations. A humanoid robot, for example, is usually expected to have robust speech recognition capabilities, and users are confused when their expectations are not met. A biomorphic form, such as a dog or cat, may be more appropriate in setting expectations about the robot's capabilities.

The social robot should mimic human-human dialogue in human-robot dialogue and be able to manage communication failures.

Social robots should recognize, respond to, and employ where possible all modalities that humans naturally use to communicate. These include verbal cues such as speech, intonation, and tone of voice, and non-verbal cues such as gesture, posture, and stance, among others. However, the robot should only communicate states it actually has. It should not fake emotions if it does not genuine use them for its own benefit. Such a fake would be detected and eventually it would be perceived negatively.

\section{The robot should mimic human social norms and be able to provide a consistent set of behaviors.}

Social robots should be aware of human social rules and norms, and grant privilege to them at all times. When possible, the robot should be aware of its own social role, its world knowledge, and what it does not know. It must be able to deal with uncertainty, and adhere to the ethical principle of least harm.

\section{Conclusion and Future Work - Designing Social Robotic Products}

In this paper, we have proposed a definition of social robots and described a framework that classifies properties of social robots. The properties include form, modality, social norms, autonomy, and interactivity. In addition, we provided guidelines for good social robot design. Based on the framework, we provide broad guidelines for the design of social robots. We envision that more specific guidelines will be generated as robots are designed and built to address specific application areas. 
Bartneck, C., \& Forlizzi, J. (2004). A Design-Centred Framework for Social Human-Robot Interaction. Proceedings of the Ro-Man2004, Kurashiki pp. 591-594. | DOI: 10.1109/ROMAN.2004.1374827

Some of the properties we proposed in our framework remain difficult to measure and hence difficult to order on the dimensions. A next step would be to define the properties in more detail and create an order of categories for the ordinal dimensions and measurements for the rational dimensions.

We invite interested readers to discuss and refine our proposed definition at this web address: http://en.wikipedia.org/wiki/Social_robot.

\section{Acknowledgements}

This work is funded by NSF IIS-0121426. Thanks to our CHI 2004 Social Robot Workshop participants for their valuable input and feedback: A.J.N. van Breemen, Carl DiSalvo, Jill Drury, Guy Hoffman, Helge Hüttenrauch, Peter Kahn and Junji Yamato. The proceedings of the workshop are available at http://www.bartneck.de/workshop/chi2004

\section{References}

Bartneck, C. (2002). Integrating the OCC Model of Emotions in Embodied Characters. Proceedings of the Workshop on Workshop on Virtual Conversational Characters: Applications, Methods, and Research Challenges, Melbourne.

Breazeal, C. (2003). Designing Sociable Robots. Cambridge: MIT Press.

Breemen, A. J. N. v. (2004). Bringing Robots To Life: Applying Principles Of Animation To Robots. Proceedings of the CHI2004 Workshop on Shaping Human-Robot Interaction - Understanding the Social Aspects of Intelligent Robotic Products, Vienna.

Brooks, R. (2002). Flesh and Machines: How Robots Will Change Us. New York: Pantheon Books.

Capek, K. (2001). R.U.R. Mineola: Dover Publications.

Condon, J. (1965). When People talk with People. In J. Condon (Ed.), Semantics and Communication (pp. 87-107). Boston: Macmillian.

DiSalvo, C., Gemperle, F., Forlizzi, J., \& Montgomery, E. (2003). The Hug: An Exploration of Robotic Form for Intimate Communication. Proceedings of the Ro-Man2003, Millbrae.
Engelhardt, K. G., \& Edwards, R. A. (1992). Human-robot integration for service robotics (Chapter 16). In M. Rahimi \& W. Karwowiski (Eds.), Human-robot interaction (pp. 315-346). New York: Taylor \& Francis.

Fong, T., Nourbakhsh, I., \& Dautenhahn, K. (2003). A survery of socially interactive robots. Robotics and Autonomous Systems, 42, 143-166.

Forlizzi, J., \& Battarbee, K. (2004). Understanding Experience in Interactive Systems. Proceedings of the DIS2004, Cambridge.

Goffman, E. (1963). Facial Engagements. In E. Goffman (Ed.), Behavior in Public Places (pp. 83-111). Boston: Macmillian.

Goza, S. M., Ambrose, R. O., Diftler, M. A., \& Spain, I. M. (2004). Telepresence Control of the NASA/DARPA Robonaut on a Mobility Platform. Proceedings of the CHI2004, Vienna.

Hall, E. T. (1964). Adumbration as a Feature of Intercultural Communication. American Anthropologist, 66(2).

Hasbro. (2004). Furby. from http://www.furby.com

Hoffman, G., \& Breazeal, C. (2004). Robots that work in collaboration with people. Proceedings of the CHI2004 Extended Abstracts, Vienna.

Hofstede, G. (1984). Culture's Consequences, International Differences in Work-Related Values. Beverly Hills: Sage Publications.

MetalForming. (1997). Tool Changers Enhance Robot Versatility - republished from MetalForming magazine. 2004, from http://www.atiia.com/qc20article1.htm

Ortony, A., Clore, G., \& Collins, A. (1988). The Cognitive Structure of Emotions. Cambridge: Cambridge University Press.

RoboticPerformanceCompany. (2004). Valerie the Robo Receptionist. from http://www.roboceptionist.com/

Sony. (1999). Aibo. from http://www.aibo.com

Trappl, R., Petta, P., \& Payr, S. (2003). Emotions in Humans and Artifacts. Cambridge: MIT Press.

UN. (2002). United Nations and the International Federation of Robotics. Proceedings of the World Robotics 2002, New York. 